\title{
Isolated Left Hepatic Duct Injury in Blunt Trauma Abdomen: Two Case Reports with Literature Review
}

\author{
Abhishek gupta, Tanmay jain, Mahakshit bhatt, Jitendra mangtani, \\ $\mathrm{K} \mathrm{K}$ dangayach \\ Mahatma gandhi hospital,jaipur
}

\begin{abstract}
Isolated extrahepatic biliary tract injury following blunt abdominal trauma is rare. The underlying pathogenic mechanisms remain obscure, but include shear and/or compression forces on the biliary system. Associated morbidity rates are high and largely the result of delays in diagnosis. . The primary aim of this paper is to describe the clinical features, diagnosis, treatment, and outcome of two patients with left hepatic duct injury after blunt abdominal trauma. As a secondary objective, a literature review is presented. The two cases presented in this study are as follows: (1) A young male, involved in a crush injury by a motor vehicle, was referred with history of blunt hepatic trauma thirty days back and abdominal distension from a general hospital. His vitals were stable. Sonography of abdomen demonstrated a large fluid collection in upper abdomen. A percutaneous drain was placed in this collection and drained approximately $5000 \mathrm{ml}$ of bile. Due to continued drainage of several hundred millilitres of bile per day an Endoscopic retrograde cholangiography (ERCP) was obtained. This demonstrated a leakage at the left hepatic duct and stenting was done. Over the next several days, the drainage markedly decreased. A repeat ERCP was conducted 12 weeks later and stent was replaced. This further decreased bilious discharge to $20 \mathrm{ml} /$ day for three days and then nil. Stent and drain was removed and patient was comfortably discharged and followed up. (2) A young male fell from a height. On exploratory laparotomy, hemoperitoneum, liver injury, retroperitoneal hematoma to the right with no biliary leak was noted at that time. $600 \mathrm{ml} /$ day bilious discharge was noted in drain from post-operative day 2. Endoscopic retrograde cholangiography was conducted 2 weeks later and revealed a large leakage at the left hepatic duct. Repeat exploratory laparotomy was performed and revealed left hepatic duct injury. Primary closure of left hepatic duct over t-tube was done. A high level of suspicion is necessary to identify injuries to the hepatic ducts. Early diagnosis that occurs during laparotomy due to associated injuries is important to reduce complications.
\end{abstract}

Keywords: blunt abdominal trauma; hepatic injury; extra hepatic bile duct injury.

\section{Introduction}

Isolated biliary tract injury following blunt abdominal injury is extremely rare [1]. The mechanism of injury remains unclear but has been attributed to shearing and/or compression forces on the extrahepatic biliary system. Morbidity rates of up to $40 \%$ have been reported, largely the result of delays in diagnosis [2-5]. Diagnostic tools useful in EHDIs include computed tomography (CT), abdominal ultrasound (AUS), nuclear imaging (HIDA), percutaneous transhepatic cholangiography (PTC), and endoscopic retrograde cholangiopancreatography (ERCP). Traditional management options in EHDI include primary repair with or without a T-tube, biliary-enteric anastomosis, ductal ligation, stenting, and drainage. Simple drainage and biliary decompression is often the most appropriate treatment option in unstable patients. Here we report 2 cases of isolated injury of left hepatic duct following blunt trauma that was successfully managed by intrabdominal drains, laparotomy and ERCP.

\section{Case Report}

1. An 18-year-old man sustained compression injury to his abdomen while working under a van. He was immediately admitted in nearby hospital where patient was kept on conservative treatment for 30 days and referred to Mahatma Gandhi Hospital. He was awake and alert with systolic blood pressure of 110 and heart rate 98. Abdominal distension and icterus was evident. Abnormal lab studies included WBC 18000, SGOT 200, SGPT 230, ALP 2000 and total bilirubin 5mg/dl. Sonography of abdomen demonstrated a large fluid collection in upper abdomen. A percutaneous drain was placed in this collection and drained approximately $5000 \mathrm{ml}$ of bile. Computerised tomography (CT) of abdomen revealed liver injury grade 3 in left lobe, free fluid in peritoneal cavity 


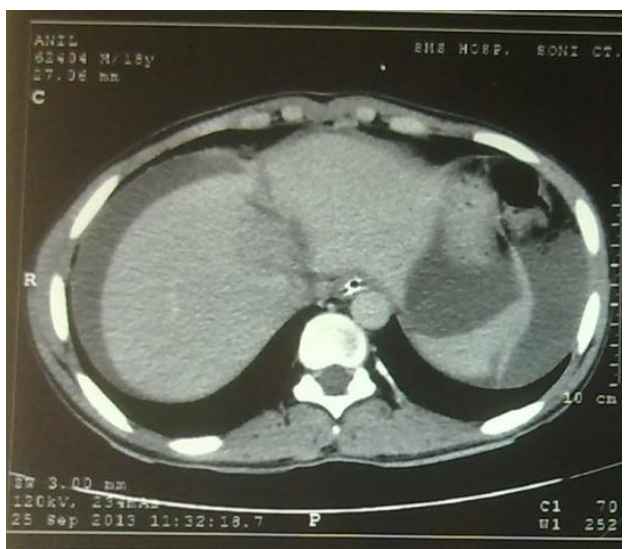

Figure 1: Computed tomographic (CT) scan showing a high-grade liver injury along with large amount of intraperitoneal fluid in the Upper abdomen. around liver and pelvis (Figure 1). ERCP revealed intrahepatic biliary leak in the left hepatic duct $3 \mathrm{~cm}$

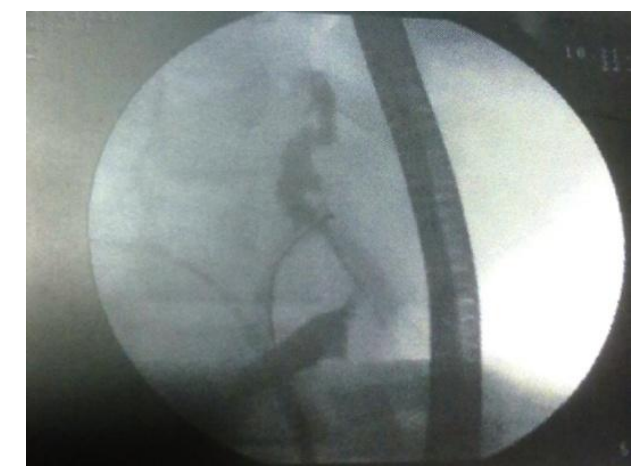

Figure 2: Initial ERCP study demonstrating left hepatic duct leak.

Above the confluence (Figure 2). Right hepatic duct was patent. 7 Fr $12 \mathrm{~cm}$ plastic DPT stent was placed across the leak. Over the next several days, the drainage markedly decreased and the patient was discharged to home with drain in place. Repeat ERCP 12 weeks after

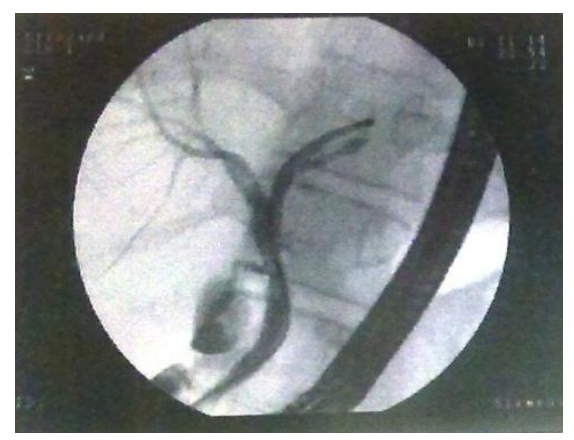

Figure 3: Repeat ERCP study demonstrating intact left hepatic duct

Patient's initial injury showed filling of both right and the left hepatic duct (Figure 3) .His liver function tests (LFTs) at the time were within normal limits. The stent and the drain were removed, with no subsequent problems reported. He is now one year out from his original trauma, has normal LFTs, and a recent abdominal sonogram showed normal common bile duct (CBD) size.

2. A 15 yr boy admitted with history of fall from height, admitted in our hospital with complains of pain in abdomen. Exploratory laparotomy revealed right perirenal haematoma with hemoperitoneum near liver, liver laceration was present with no active bleeding or biliary leak at the time of operation and a Jackson-Pratt drain was left in right upper quadrant. The patient's early hospital course was uneventful. However, he subsequently began draining increasing amounts of bile from his abdominal drain, associated with concurrent rise in bilirubin. Patient underwent ERCP 1 week later which revealed high grade biliary leak from 


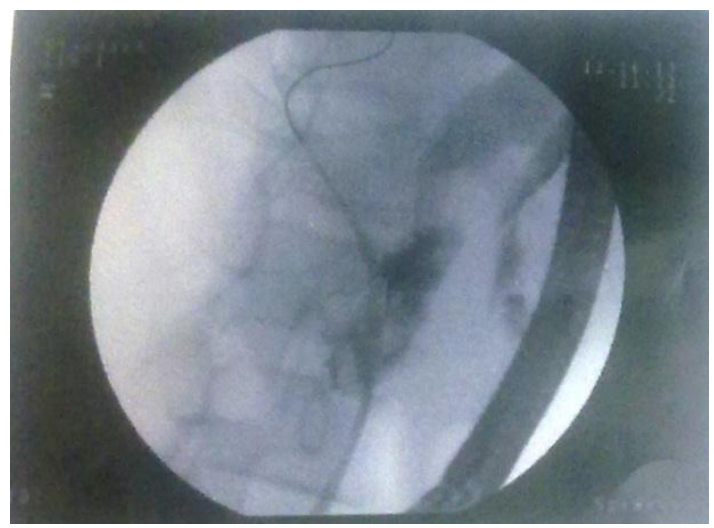

Figure 4: left hepatic duct leak

left hepatic duct (Figure 4). Right hepatic duct was patent. Sphincterotomy done. 7 Fr $12 \mathrm{~cm}$ DPT plastic stent was placed above the leak into the right system. Patient improved initially and drain output reduced to $200 \mathrm{ml}$ per day but 3 days after stenting patient developed signs of biliary peritonitis and was planned for laparotomy. Exploration revealed left hepatic duct injury for which primary closure of left hepatic duct over ttube was done and drain was put in right upper quadrant. The daily drain output subsequently reduced. A T-tube cholangiogram was performed on $10^{\text {th }}$ post-operative day which showed normal filling of both ducts with no leakage of contrast. Both T-tube and drain were removed with no subsequent problems were reported.

\section{Discussion}

Bile duct injuries following blunt trauma abdomen are rare. Rodriguez and parks reported an incidence of 0.37 and $0.5 \%$ respectively [1-4].

Bourque et al in their review in 1989 reported only 125 cases of damage to the extrahepatic biliary system after blunt trauma abdomen since 1806.most cases consist of gall bladder injury making damage to the bile ducts even rarer. The first case of bile duct injury was published in 1799 by Wainwright who described it in a post- mortem examination on a man who fell from his horse [6]. Dawson et al found only 24 cases of damage to the extra-hepatic bile ducts after blunt trauma in their 1991 review.

Damage to the bile ducts is usually part of a combined injury, which most frequently involves the liver. To our best knowledge isolated left bile duct injury has only been described in literature thrice before [7, 8, and 9]. The diagnosis of biliary leakage is often missed at primary survey. In 32 of the 44 cases in the literature there was a diagnostic delay of 1 day to 5 weeks. In 14 of the last 20 cases there was a delay with a median of 7 days. Even when an exploratory laparotomy is performed up to $36 \%$ of bile duct lesions go undetected[10]. It is therefore recommended that, if there is a high index of suspicion, visualisation of the bile ducts by means of a cholangiography should be part of the exploration. Delayed presentation includes symptoms like jaundice, abdominal distension, abdominal pain, vomiting and fever. Bile duct lesions may initially go undetected on ultrasound and CT because the leakage of bile is only detected when a certain amount of fluid has accumulated. If there is a high suspicion of bile duct injury, a HIDA-scan or ERCP is recommended [10, 11, 12, and 13]. ERCP has the additional advantage that it can be therapeutic, i.e. by means of insertion of an endoprothesis. MRCP is probably less sensitive in detecting lesions and lacks the therapeutic advantage. If no emergency laparotomy is indicated stenting of the lesion by means of ERCP or PTC are safe and minimal invasive techniques. If there is only a small leak simple drainage of the bile also leads to healing of the defect. If the lesion is partial and preferably less than $50 \%$ of the circumference of the duct primary suture is the therapy of choice when a laparotomy is indicated. However, if a total rupture exists a hepatico- or choledocho-jejunostomy should be performed. Primary suture in these cases will very likely lead to stricturing and narrowing of the anastomosis. When the diagnosis of EHDIs occurs during an emergent celiotomy, the primary focus should be on patient stabilization, haemostasis, temporizing stenting, ligation, and T-tube placement should be considered .When there is an intrahepatic bile duct lesion combined with infection or large devitalisation of the hepatic parenchyma a partial hepatectomy should be performed.

\section{Conclusion}

Traumatic bile duct lesions are very rare. Because the clinical presentation is often insidious, extra hepatic bile duct injuries are frequently missed on the initial clinical evaluation. The management of extra hepatic duct injuries has changed over the last decade. If there is a high index of suspicion the biliary system should always be visualised. With good short-term results of ERCP being well established, a growing body of data is demonstrating equally good results on long-term follow up. If a lesion consists of less than $50 \%$ of the circumference of the duct primary suture or stenting are the best therapeutic options. Late recognition and 
inappropriate management of these injuries result in severe, often fatal consequences. Biliary drainage and control of sepsis should be the initial goals of treatment. Definitive surgical reconstruction should be delayed for several months.

\section{References}

[1]. R. W. Parks and T. Diamond, "Non-surgical trauma to the extrahepatic biliary tract," British Journal of Surgery, vol. 82, no. 10, pp. $1303-1310,1995$.

[2]. M. W. Hills, A. J. Richardson, N. Tait, S. A. Deane, and J. M. Little, "Non-iatrogenic trauma to the extrahepatic biliary tract," Australian and New Zealand Journal of Surgery, vol. 63, no. 3, pp. 190-194, 1993

[3]. Ivatury RR, Rohman M, Nallathambi M, Rao PM, Gunduz Y, Stahl WM. The morbidity of injuries of the extra-hepatic biliary system. J Trauma. 1985;25:967-973.

[4]. J. A. Rodriguez-Montes, E. Rojo, and L. García-Sancho Martín, "Complications following repair of extrahepatic bile duct injuries after blunt abdominal trauma," World Journal of Surgery, vol. 25, no. 10, pp. 1313-1316, 2001.

[5]. M. D. Bourque, N. Spigland, A. L. Bensoussan, and L. H. Garel Blanchard, "Isolated complete transection of the common bile duct due to blunt trauma in a child, and review of the literature," Journal of Pediatric Surgery, vol. 24, no. 10, pp. 1068-1070, 1989

[6]. Wainwright T. Letter. Med Phys J 1799; 362-4.

[7]. Salam M, Glowniak JV, Vetto RM, et al. Detection of bile leakage from traumatic right hepatic duct laceration with Technetium 99m DISIDA cholescintigraphy. Clin Nucl Med 1987; 12(8):589_-91.

[8]. Michelassi F, Ranson JHC. Bile duct disruption by blunt trauma. J Trauma 1985; 25:45-454.

[9]. A.G.J. van Marle a,*, H. van Overhagen b, J.J. Nicolai c, M.A.M. Brouwers. Minimal invasive therapy of an isolated rupture of the right hepatic duct after blunt abdominal trauma. Injury extra 2006;37;211-217

[10]. Veroux M, Cillo U, Madia C, Veroux P, Gringeri E, Fiamingo P, et al. Traumatic complete transection of the left hepatic duct: another approach to repair. Eur J Surg 2002; 168(10): 563-5.

[11]. SandersDW, Andrews DA. Conservative management of hepatic duct injury after blunt trauma: a case report. J Pediatric Surg 2000; 35(10): $1503-5$.

[12]. Sakamoto Y, Tanaka N, Furuya T, Okamoto H, Nagai M, Murakawa T, et al. A simple stenting method for management of hepatic ductal injury secondary to blunt abdominal trauma: two case reports. J Pediatr Surg 2000; 35(10):1503-5.

[13]. Bawa AS, Attri AK, Motup T, Kaushik R. Isolated right hepatic duct injury following blunt abdominal trauma: case report. Trop Gastroenterol 2001; 22(1):49-50. 